\title{
Effect of microRNA-144-5p on the proliferation, invasion and migration of human umbilical vein endothelial cells by targeting SMAD1
}

\author{
WEI FU, ZIDONG LIU, JING ZHANG, YUXUE SHI, RUIYAO ZHAO and HENG ZHAO \\ Department of Cardiology, The Third Affiliated Hospital of Jinzhou Medical University, \\ Jinzhou, Liaoning 121000, P.R. China
}

Received December 11, 2018; Accepted August 21, 2019

DOI: $10.3892 /$ etm.2019.8194

\begin{abstract}
Atherosclerosis is a multifactorial chronic disease that is a major cause of death and injury worldwide. Apoptosis of endothelial cells (ECs) serves an important role in the occurrence and development of atherosclerosis. MicroRNAs (miRNAs) serve a key role in atherosclerosis though regulating the function of ECs. At present, the role of miRNA-144-5p (miR-144-5p) in atherosclerosis is unclear. The aim of this study was to investigate the effect of miR-144-5p on atherosclerosis in oxidized low-density lipoprotein (ox-LDL)-stimulated human umbilical vein endothelial cells (HUVECs). Results from the present study demonstrated that miR-144-5p overexpression could inhibit proliferation and induce apoptosis in HUVECs. To further study the biological function of miR-144-5p, the effects of modulating miR-144-5p expression on the invasion and migration of HUVECs were also examined. The results demonstrated that miR-144-5p upregulation suppressed HUVEC migration and invasion. TargetScan and dual luciferase reporter assay results demonstrated that SMAD1 was a direct target gene of miR-144-5p. miR-144-5p upregulation inhibited the expression of phosphorylated-SMAD1/5/8 in the SMAD pathway. In conclusion, the data indicated that miR-144-5p serves an important role in the development of atherosclerosis through regulating the function of HUVECs by targeting SMAD1.
\end{abstract}

\section{Introduction}

Atherosclerotic cardiovascular disease and its clinical complications, such as myocardial infarction and ischemic stroke, are the world's first and third causes of death, respectively, causing

Correspondence to: Dr Zidong Liu, Department of Cardiology, The Third Affiliated Hospital of Jinzhou Medical University, 2 Heping Road, Linghe, Jinzhou, Liaoning 121000, P.R. China

E-mail: liuzd181201@163.com

Key words: atherosclerosis, microRNA-144-5p, HUVEC, SMAD1, SMAD pathways
247.9 deaths $/ 100,000$ persons in 2013 , representing $84.5 \%$ of cardiovascular deaths and $28.2 \%$ of all-cause mortality (1). Atherosclerosis can lead to lipid accumulation, extracellular matrix protein deposition, and calcification in the intima and media of the arteries causing arterial stiffness and reducing arterial elasticity (2). Oxidized low-density lipoprotein (ox-LDL) is able to induce apoptosis of endothelial cells (ECs) and is considered as the major risk factors for atherosclerosis ((3). In response to oxidized low-density lipoproteins (oxLDL), endothelial cells express a range of chemokines and adhesion molecules that contribute to leukocyte recruitment, adherence, and migration into the subendothelium (4), the first stage in the development of atherosclerosis (5). Recently, increasing evidence has indicated that microRNAs (miRNAs) serve an important role in atherosclerosis development and progress (6).

miRNAs, widely found in plants and animals, including humans, are a group of non-coding, single-stranded RNA molecules that participate in sequence-specific post-transcriptional regulation of gene expression (7-9). miRNAs have emerged as crucial players in many biological processes, and changes in their expression or function are associated with numerous human diseases (10). It has been reported that miRNAs regulate several cellular and molecular biological processes related to the development of atherosclerosis ranging from interacting with risk factors to initiating the development, promoting the progression and causing the rupture of atherosclerotic plaques (11). miRNA (miR)-144-5p has been studied in several diseases, including cancer (12-14), chronic periodontitis (15) and depressive disorders (16). However, the specific function and mechanism of action of miR-144-5p in atherosclerosis remain unclear.

SMAD proteins are intracellular mediators of the transforming growth factor- $\beta$ family. Smad1/5/8 play an important role in angiogenesis. Phosphorylation and activation of the transcription factors SMAD1/5/8 results in the promotion of angiogenesis. The SMAD1/5/8 signaling pathway, which inhibits extracellular matrix deposition, promotes endothelial cell proliferation and migration (17). A previous study has reported that the balance of proliferation/apoptosis of vascular smooth muscle cells in atherosclerosis is modulated by long non-coding RNA-MEG3 via the regulation of the miR-26a/Smad1 axis (18). In addition, activation of CD137 signaling promotes 
angiogenesis in atherosclerosis by modulating the endothelial Smad1/5-nuclear factor of activated $T$ cells pathway (19). In a previous study using bioinformatics, it was predicted that SMAD1 was a direct target gene of miR-144-5p (20).

The present study aimed to investigate the role of miR-144-5p in atherosclerosis and to further explore the molecular mechanism of action of miR-144-5p. It was hypothesized that miR-144-5p alleviates ox-LDL-induced HUVEC proliferation, invasion and migration by targeting SMAD1, and further implicates the potential therapeutic targets to reverse atherosclerosis.

\section{Materials and methods}

Cell culture and cell transfection. HUVECs were acquired from the Shanghai Institute of Life Sciences, Chinese Academy of Sciences. Culture media for HUVECs contained routine medium 199 (Gibco; Thermo Fisher Scientific, Inc.) with 10\% FBS (unless otherwise stated) (Gibco; Thermo Fisher Scientific, Inc.), $1 \%$ penicillin/streptomycin (Gibco; Thermo Fisher Scientific, Inc.), $1 \%$ endothelial cell growth supplement (Sigma-Aldrich; Merck KGaA) and $10 \mathrm{ng} / \mathrm{ml}$ epidermal growth factor in a $5 \% \mathrm{CO}_{2}$ humidified atmosphere at $37^{\circ} \mathrm{C}$. To mimic atheroprone conditions, HUVECs were exposed to proatherogenic oxLDL ( $25 \mu \mathrm{g} / \mathrm{ml}$; Biomedical Technologies S.L.).

HUVECs $\left(5.0 \times 10^{4}\right.$ cells/well) were transfected with $100 \mathrm{nM}$ miR-144-5p mimic or $100 \mathrm{nM}$ mimic control for 48 hat $37^{\circ} \mathrm{C}$ using Lipofectamine $^{\circledR} 2000$ (Invitrogen; Thermo Fisher Scientific, Inc.) according to the manufacturer's instructions. After $48 \mathrm{~h}$ of transfection, the transfection efficiency was detected using reverse transcriptase quantitative PCR (RT-qPCR).

MTT assay. Cell proliferation was detected by MTT assay. HUVECs $\left(2.0 \times 10^{3}\right.$ cells/well) were plated into 96-well plates and cultured at $37^{\circ} \mathrm{C}$ for 12,24 or $48 \mathrm{~h}$, then $20 \mu \mathrm{l}$ MTT ( $5 \mathrm{mg} / \mathrm{ml}$; Sigma-Aldrich, Merck KGaA) was added to medium. Following 4-h incubation, $150 \mu \mathrm{l}$ DMSO was used to dissolve the formazan crystals. The absorbance was measured at a wavelength of $490 \mathrm{~nm}$ using a microplate reader.

Dual-luciferase reporter assay. TargetScan (v7.2) bioinformatics software (http://www.targetscan.org/vert_72/) was used to predict target genes of miR-144-5p. The 3'-untranslated region (UTR) of SMAD1 was cloned into the luciferase reporter vector psiCHECK-2 (Promega Corporation) according to the manufacturer's instruction. Briefly, $500 \mathrm{ng}$ of each reporter construct [wild-type (WT) or mutant 3'-UTR of SMAD1 or the psiCHECK-2 vector] and miR-144-5p mimic or mimic control were co-transfected into pre-confluent (60-70\%) HUVECs plated in 24-well plate using Lipofectamine 2000 for $48 \mathrm{~h}$ at $37^{\circ} \mathrm{C}$. Then relative luciferase activity was detected with a microplate reader (Molecular Devices, LLC). Renilla luciferase was used for normalization. Each sample was repeated three times.

Wound healing assay. To detect the migratory ability of HUVECs, wound healing assays were performed. HUVECs ( $3 \times 10^{5}$ per well) were seeded in 6 -well plates with human endothelial serum-free culture medium (cat. no. 11111044; Gibco; Thermo Fisher Scientific, Inc.) supplemented with 0.5\% FBS and cultured until $90 \%$ confluent; the cell monolayer was subsequently scraped with a pipette tip. The cells were washed three times with PBS to wipe off cell debris and the cells were cultured in human endothelial serum-free culture medium without FBS. Images were captured at 0 and $24 \mathrm{~h}$ using a Olympus IX71 Inverted Microscope (Olympus Corporation; magnification, $\mathrm{x} 100)$.

Transwell invasion assay. Transwell invasion assays were performed with using a Transwell chamber $(8 \mu \mathrm{m}$ pore size; Corning, Inc.). Cells $\left(2 \times 10^{4}\right.$ cells/well $)$ were collected and re-suspended in human endothelial serum-free culture medium (cat. no. 11111044; Gibco; Thermo Fisher Scientific, Inc.) containing $0.5 \%$ FBS and plated on the top of polycarbonate Transwell filter pre-coated with Matrigel (BD Biosciences). Complete culture medium (DMEM, Gibco; Thermo Fisher Scientific, Inc.) containing 10\% FBS was added to the lower chamber. After incubation for $24 \mathrm{~h}$ at $37^{\circ} \mathrm{C}$, the non-invasive free cells were carefully removed with cotton swab from the top chamber. The invaded cells on the lower membranes were fixed with $4 \%$ paraformaldehyde for $20 \mathrm{~min}$ at room temperature and stained by $0.1 \%$ crystal violet for $15 \mathrm{~min}$ at room temperature. Cells were counted in five random fields for each chamber at a magnification of x 100 under Olympus IX71 Inverted Microscope (Olympus Corporation) to evaluate invasive capacity.

$R T-q P C R$. Total RNA was extracted from HUVECs $\left(5 \times 10^{4}\right.$ cells/well) using TRIzol ${ }^{\circledR}$ (Invitrogen; Thermo Fisher Scientific, Inc.) according to the manufacturer's instructions. To determine the expression levels of miR-144-5p and SMAD1 mRNA, cDNA was synthesized using PrimeScript Reverse Transcriptase Reagent kit (Takara Biotechnology Co., Ltd.) according to the manufacturer's instruction. qPCR was performed with the SYBR Premix Ex Taq kit (Takara Biotechnology Co., Ltd.) according to the manufacturer's protocol. U6 and GAPDH were used as the endogenous controls. The thermocycling conditions were as follows: $95^{\circ} \mathrm{C}$ for $5 \mathrm{~min}$, followed by 38 cycles of denaturation at $95^{\circ} \mathrm{C}$ for $15 \mathrm{sec}$ and annealing/elongation at $60^{\circ} \mathrm{C}$ for $30 \mathrm{sec}$. The primers used were as follows: U6, forward 5'-GCTTCGGCAG CACATATACTAAAAT-3', reverse 5'-CGCTTCACGAATTT GCGTGTCAT-3'; GAPDH, forward 5'-CTTTGGTATCGTG GAAGGACTC-3', reverse 5'-GTAGAGGCAGGGATGATGT TCT-3'; SMAD1, forward 5'-ACAGTCTGTGAACCATGGAT TTGA-3', reverse 5'-TGAGGTGAACCCATTTGAGTAA GAA- 3 '. The experiments were repeated in triplicate, and the $2^{-\Delta \Delta C q}$ method was used to calculate the relative gene expression and normalized to the internal reference gene U6 or GAPDH, respectively (21).

Western blotting. HUVECs $\left(5 \times 10^{4}\right.$ cells/well) were lysed using RIPA Lysis Buffer (Gibco; Thermo Fisher Scientific, Inc.) with protease inhibitor PMSF (Shanghai Biocolor BioScience \& Technology Co., Ltd.). BCA Protein Assay kit (Thermo Fisher Scientific, Inc.) was used to measure protein concentrations. A total of $20 \mu \mathrm{g}$ of protein was subjected to $10 \%$ SDS-PAGE electrophoresis and transferred to PVDF membranes. The membranes were blocked with 5\% Difco ${ }^{\mathrm{TM}}$ skim milk (cat. no. 232100, BD Biosciences) at room temperature for $1 \mathrm{~h}$. Subsequently, the membranes were probed with primary antibodies against SMAD1 (cat. no. 6944; 1:1,000; Cell Signaling Technology, Inc.), phosphorylated (p)-SMAD1 (cat. no. 5753; 1:1,000; Cell 

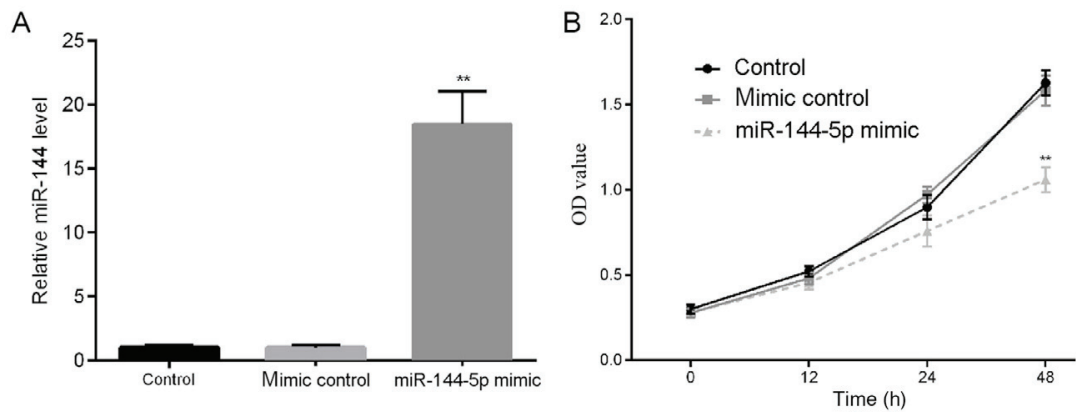

C
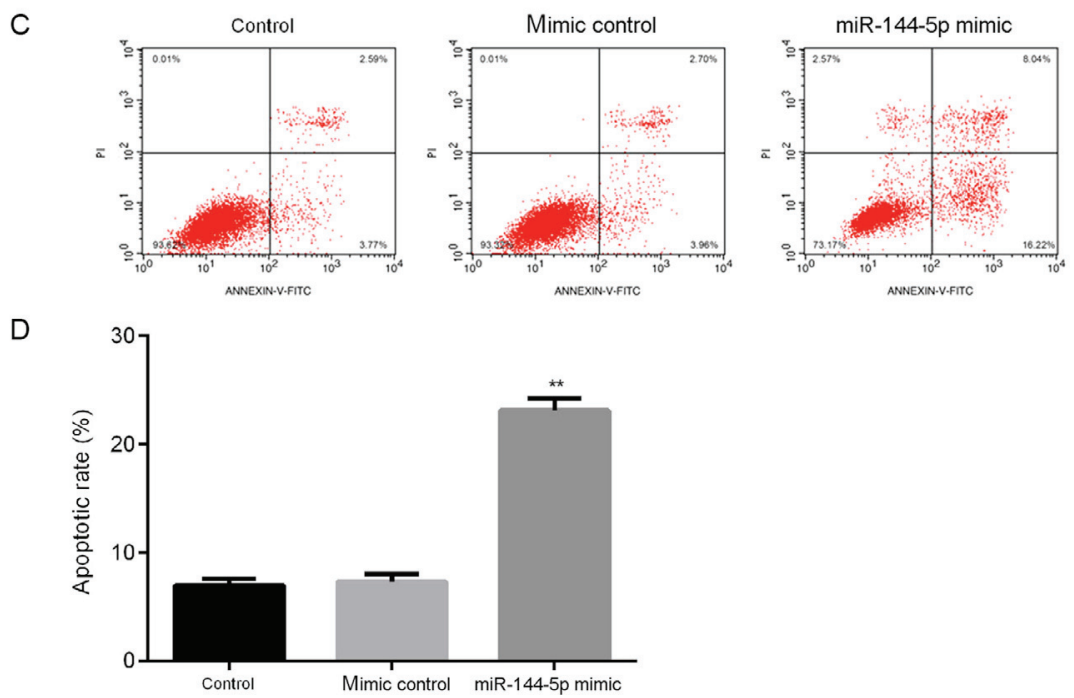

Figure 1. miR-144-5p mimic suppresses cell viability and promotes apoptosis in HUVECs. HUVECs were transfected with mimic control and miR-144-5p mimics for $48 \mathrm{~h}$. (A) RT-qPCR was used to detect relative expression level of miR-144-5p. (B) MTT assay was performed to analyze cell viability. (C) Flow cytometry was performed to detect cell apoptosis and (D) apoptotic rates were calculated at 12,24 and $48 \mathrm{~h}$. ${ }^{* *} \mathrm{P}<0.01 \mathrm{vs.}$ control. miR-144-5p, microRNA-144-5p; OD, optical density; PI, propidium iodide; RT-qPCR, reverse transcriptase quantitative PCR.

Signaling Technology, Inc.), SMAD5 (cat. no. 12534; 1:1,000; Cell Signaling Technology, Inc.), p-SMAD5 (cat. no. ab92698; 1:1,000; Abcam), SMAD8 (cat. no. sc-293413; 1:1,000; Santa Cruz Biotechnology, Inc.), p-SMAD8 (cat. no. sc-12353, 1:1,000; Santa Cruz Biotechnology, Inc.), and GAPDH (cat. no. 5174; 1:1,000; Cell Signaling Technology, Inc.), at $4^{\circ} \mathrm{C}$ overnight. The next day, the membrane was washed three times with PBS-Tween-20 (0.05\%) buffer and then incubated with horse radish peroxidase (HRP)-conjugated goat anti-rabbit secondary antibody (cat. no. 7074; 1:1,000; Cell Signaling Technology, Inc.) or mouse $\operatorname{IgG\kappa }$ light chain binding protein-HRP (cat. no. sc-516102; 1:1,000; Santa Cruz Biotechnology, Inc.) for $1 \mathrm{~h}$ at room temperature. The residue antibody solution was completely washed off with PBST, and protein bands were visualized using RapidStep ${ }^{\mathrm{TM}}$ ECL Reagent (cat. no. 345818; Merck KGaA). GAPDH served as loading control for normalization. Blot bands were semi-quantitively analyzed using ImageJ version 4.0 (National Institutes of Health).

Flow cytometry apoptosis assay. The Annexin V-FITC Apoptosis Detection kit I (BD Biosciences) was used to detect cell apoptosis, according to the manufacturer's protocol. A total of 100,000 suspended cells were washed twice with PBS, collected, centrifuged with $1,000 \times \mathrm{g}$ for $5 \mathrm{~min}$ at $20^{\circ} \mathrm{C}$, and re-suspended in $100 \mu \mathrm{l}$ of FITC-binding buffer. Subsequently, $\sim 5 \mu 1$ ready-to-use Annexin V-FITC (BD Biosciences) and $5 \mu \mathrm{l}$ propidium iodide (PI) were added. Cells were incubated for $30 \mathrm{~min}$ at room temperature in the dark. Annexin V-FITC and PI fluorescence were assessed by BD FACSCalibur flow cytometer (BD Biosciences). The percentages of cells in early apoptosis (Annexin $\mathrm{V}^{+} / \mathrm{PI}^{-}$) and late apoptosis (Annexin $\mathrm{V}^{+} / \mathrm{PI}^{+}$) were calculated.

Statistical analysis. All data are presented as the mean \pm SD from three independent experiments in triplicate. Student's t-test or one-way ANOVA followed by Tukey's post hoc test was used for biostatistical analysis, using SPSS 19.0 (IBM Corp.). $\mathrm{P}<0.05$ was considered to indicate a statistically significant difference.

\section{Results}

Effects of miR-144-5p on HUVEC proliferation and apoptosis. Apoptosis of endothelial cells serves an important role in the occurrence and development of atherosclerosis (22). To study the role of miR-144-5p in atherosclerosis, firstly the effects of miR-144-5p on HUVEC proliferation and apoptosis were explored. HUVECs were transfected with mimic control or miR-144-5p mimic for $48 \mathrm{~h}$. RT-qPCR was used to detect the transfection efficiency, and the results demonstrated that compared with the control group, transfection with miR-144-5p mimic significantly increased the level of miR-144-5p in HUVECs (Fig. 1A). Results from the MTT assay demonstrated that miR-144-5p mimic transfection 
A
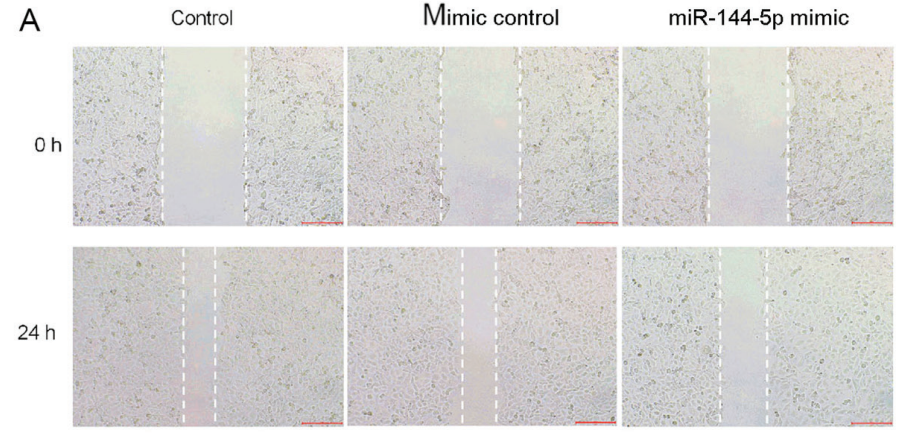

B

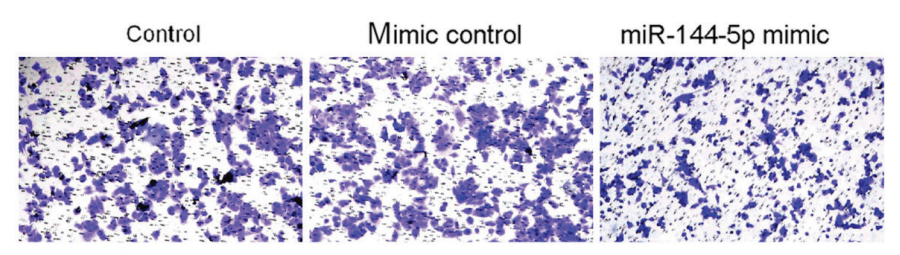

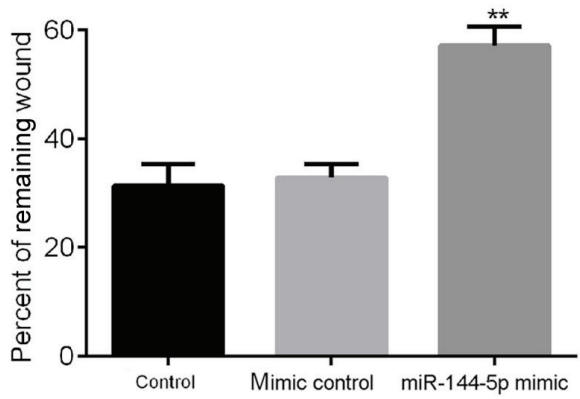

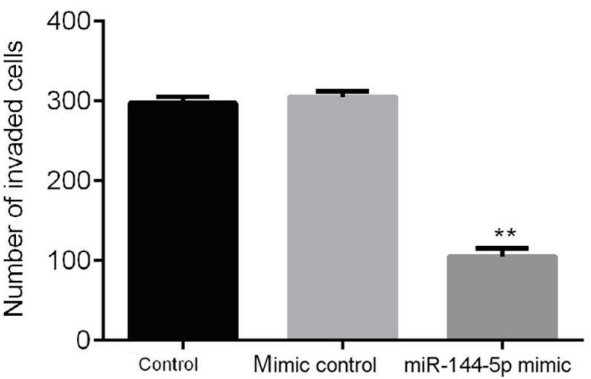

Figure 2. miR-144-5p mimic suppresses HUVEC migration and invasion. (A) Wound healing assays were performed to detect the migratory abilities of HUVECs, and the percent of remaining wound was presented (\% compared with $0 \mathrm{~h}$ ). (B) Matrigel assays were used to analyze the invasive abilities of the cells. ${ }^{* *} \mathrm{P}<0.01$ vs. control. miR-144-5p, microRNA-144-5p.

A

WT SMAD1 3'UTR

hsa-miR-144-5p

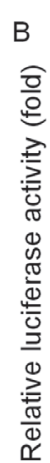

5'- UUGGUAACAUACUGUUGAUAUCA-3'

3'- gaAugucauauacuacuauagg-5'

5'- UUGGUAACAUACUGUCCGCGCAA-3'

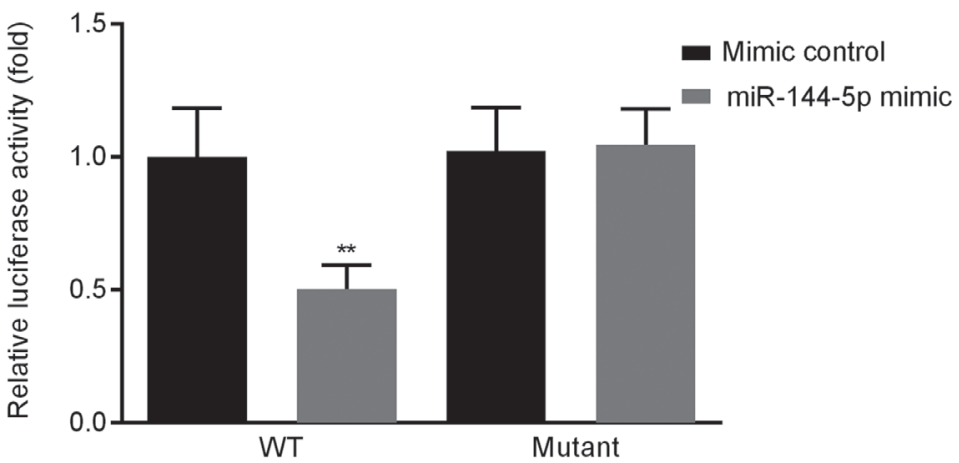

Figure 3. SMAD1 is the direct target gene of miR-144-5p. (A) TargetScan was used to predict SMAD1 as a putative target gene of miR-144-5p. (B) Dual-luciferase reporter assays were performed to confirm the relationship between miR-144-5p and SMAD1. ${ }^{* *} \mathrm{P}<0.01$ vs. mimic control. miR-144-5p, microRNA-144-5p; UTR, untranslated region; WT, wild-type.

reduced cell proliferation of HUVECs (Fig. 1B). In addition, to determine whether miR-144-5p regulated the apoptosis of HUVECs, flow cytometric analyses were performed to detect cell apoptosis. The results indicated that miR-144-5p mimic transfection significantly increased the apoptotic rates of HUVECs compare with the control (Fig. 1C and D).

Effects of miR-144-5p on HUVEC migration and invasion. To further examine the effects of miR-144-5p on the biological function of HUVECs, wound healing and Matrigel assays were performed (Fig. 2). The wound healing assay results demonstrated that miR-144-5p mimic suppressed cell migration compared with control groups (Fig. 2A). Matrigel assay results indicated that miR-144-5p mimic significantly inhibited the invasive ability of HUVECs (Fig. 2B). Taken together, these results showed that miR-144-5p inhibited the invasive and migratory potential of HUVECs.

SMAD1 is the direct target gene of miR-144-5p. To investigate the underlying mechanism of miR-144-5p on HUVECs, a putative biological target gene of miR-144-5p was identified and verified; the online bioinformatics tool TargetScan was used to predict target genes. The results identified a miR-144-5p target site in the SMAD1 3'UTR (Fig. 3A). Dual-luciferase reporter 

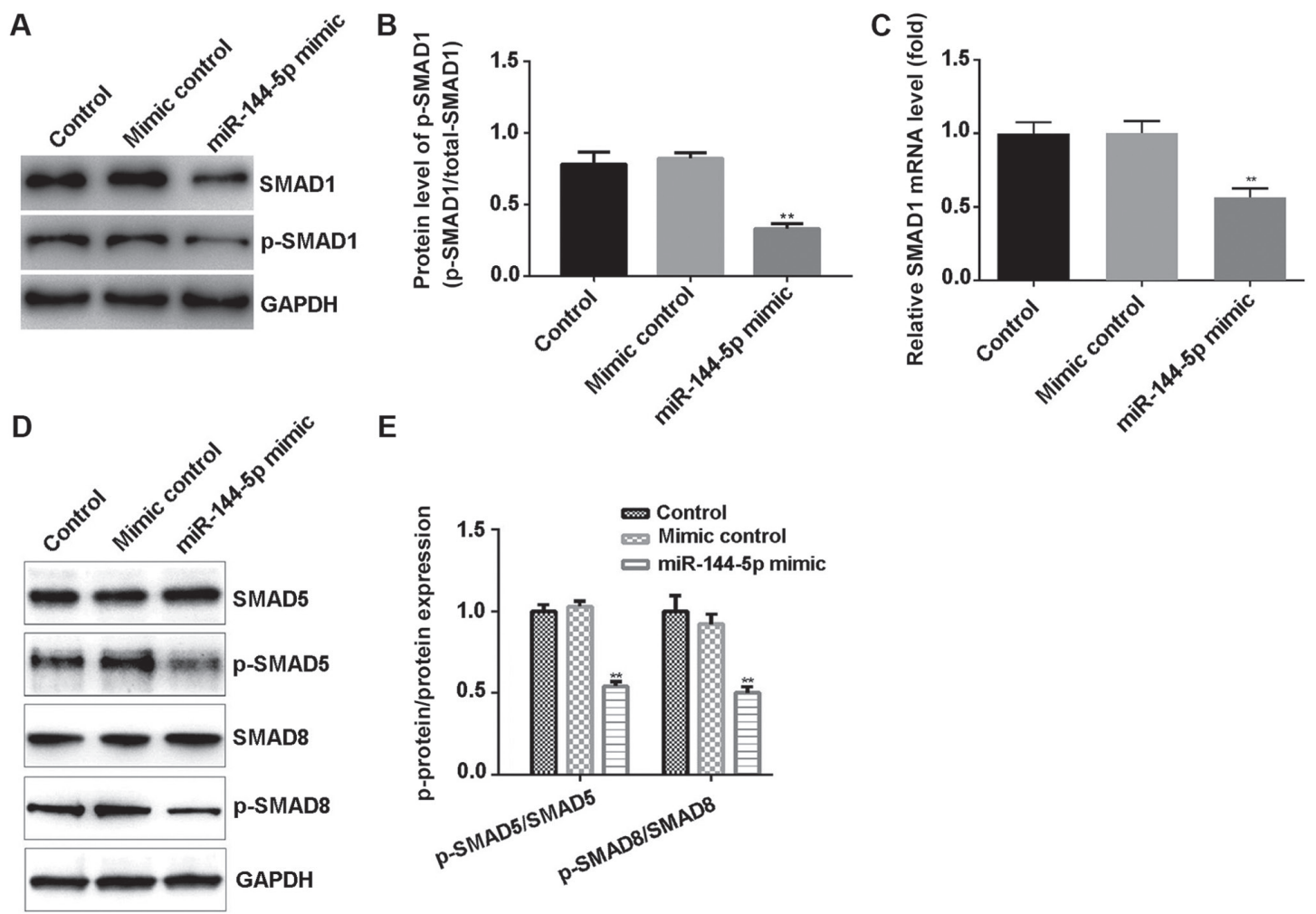

$\mathbf{E}$

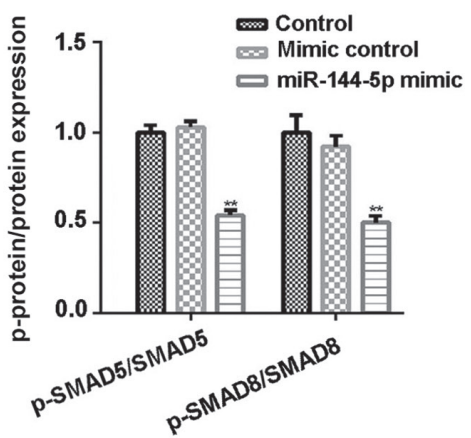

Figure 4. miR-144-5p mimic inhibits the expression of p-SMAD1/5/8. HUVECs were transfected with mimic control and miR-144-5p mimics for $48 \mathrm{~h}$. (A and B) The protein expression levels of p-SMAD1 and SMAD1 were detected by western blotting, and p-SMAD1/SMAD1 was calculated. (C) The relative mRNA expression levels of SMAD1 was detected by reverse transcriptase quantitative PCR assay. (D and E) The protein expression levels of p-SMAD5/8 and SMAD5/8 were detected by western blotting, and p-SMAD5/SMAD5 and p-SMAD8/SMAD8 was calculated. ${ }^{* *} \mathrm{P}<0.01$ vs. mimic control. miR-144-5p, microRNA-144-5p; p, phosphorylated.

assay results revealed that miR-144-5p mimic reduced the luciferase activity of the WT SMAD1 3' UTR, but miR-144-5p mimic did not inhibit that of the reporter fused to the mutant SMAD1 3' UTR (Fig. 3B). Taken together, these results demonstrated that SMAD1 was a direct target gene of miR-144-5p.

miR-144-5p inhibited SMAD1/5/8 pathway in HUVECs. To investigate the specific mechanism of action of miR-144-5p on HUVECs, the expression levels of proteins within the SMAD1/5/8 signaling pathway were examined. Western blot assay suggested that miR-144-5p mimic markedly decreased the protein level of SMAD1 and p-SMAD1 (Fig. 4A), and significantly reduced the ratio of $\mathrm{p}-\mathrm{SMAD} 1 / \mathrm{SMAD} 1$ (Fig. 4B). RT-qPCR assay results showed that miR-144-5p mimic decreased the relative mRNA expression of SMAD1 in HUVECs (Fig. 4C). Moreover, western blotting indicated that miR-144-5p mimic significantly decreased p-SMAD5/8 protein expression in HUVECs (Fig. 4D and E).

\section{Discussion}

In this present study, the primary aim was to explore the effect of miR-144-5p on HUVECs in vitro, to determine the role of miR-144-5p in atherosclerosis. It was demonstrated that miR-144-5p mimic transfection could suppress proliferation and induce apoptosis in HUVECs. It was also found that miR-144-5p mimic suppressed HUVEC migration and invasion. Furthermore, it was found that SMAD1 was a direct target of miR-144-5p, and miR-144-5p mimic suppressed the SMAD1/5/8 pathway in HUVECs.

miRNAs are a group of small, non-coding RNAs (9). Recent reports have identified multiple miRNAs that are involved in the pathogenesis of atherosclerosis (23-29). Vascular endothelial cells are crucial barriers in the vascular lumen (30). They maintain the stability of hemodynamics and material exchange, secrete inflammatory cytokines and regulate blood pressure through vasodilation and contraction factors (31). Previous studies have demonstrated that various miRNAs have different roles in the injury of vascular endothelial cells in both normal and tumor tissues. For example, previous research indicated that miRNA-129-1 and miRNA-133 promote vascular endothelial cell proliferation (32). However, the specific function and mechanism of action of miR-144-5p on HUVECs remain unclear. Consistent with these previous results, the present study provided evidence supporting the important role of miR-144-5p in HUVECs; the data demonstrated that miR-144-5p mimic suppressed the proliferation, migration and invasion of HUVECs and induced cell apoptosis.

The pathological role of miR-144-5p was further explored by identifying its direct target gene. Through bioinformatics, it was predicted that SMAD1 was a direct target of miR-144-5p. SMAD1 is a member of the SMAD family, and the family members are signal transducers and transcriptional modulators that mediate multiple signaling pathways (20). SMAD1 mediates bone morphogenetic proteins (BMP) signaling, and BMP stimulation increases SMAD1 phosphorylation, 
allowing it to form complexes with GAL4 to act as a functional transcription regulator (33). The BMP-SMAD1/5/8 signaling pathway participates in multiple biological processes including cell growth, apoptosis, morphogenesis, differentiation and immune modulation. A previous study also reported that SMAD1 is a target gene of miR-144 (20). Ren et al reported that miR-144 upregulation could decrease the activity of mTOR signaling pathways and suppress cell proliferation in osteosarcoma cells (34). In the present study, it was found that miR-144-5p was associated with the SMAD signaling pathway. miR-144-5p mimic inhibited the expression of p-SMAD1/5/8. Consequently, these results further confirm that miR-144-5p serves a crucial role in HUVECs.

In conclusion, miR-144-5p may modulate HUVECs proliferation, apoptosis, invasion and migration through affecting the SMAD signaling pathway by altering the expression of SMAD1, and thus may participate in the onset and development of atherosclerosis. Therefore, the data from this present study may provide a new theoretical basis and strategy for the diagnosis and treatment of atherosclerosis. However, this study is only a preliminary investigation in to the role of miR-144-5p in atherosclerosis. Further studies are needed to better understand the role of miR-144-5p in atherosclerosis. For example, it would be interesting to investigate whether SMAD1 upregulation could reverse the effect of miR-144-5p on HUVECs. How the SMAD1/5/8 pathway is involved in the effect of miR-144-5p in HUVECs should also be further explored. Furthermore, the effect of downregulating miR-144-5p in HUVECs should investigated. Finally, the relationship between the expression of miR-144-5p and SMAD1, in the context of the clinical features of atherosclerosis needs to be explored.

\section{Acknowledgements}

Not applicable.

\section{Funding}

No funding was received.

\section{Availability of data and materials}

The datasets used and/or analyzed during the current study are available from the corresponding author on reasonable request.

\section{Authors' contributions}

ZL designed the study and revised the manuscript. WF and JZ wrote the manuscript and collected the data. YS and RZ searched the literature and interpreted the data. HZ collected the data. All authors read and approved the final manuscript.

\section{Ethics approval and consent to participate}

Not applicable.

\section{Patient consent for publication}

Not applicable.

\section{Competing interests}

The authors declare that they have no competing interests.

\section{References}

1. Ference BA, Ginsberg HN, Graham I, Ray KK, Packard CJ, Bruckert E, Hegele RA, Krauss RM, Raal FJ, Schunkert H, et al: Low-density lipoproteins cause atherosclerotic cardiovascular disease. 1. Evidence from genetic, epidemiologic, and clinical studies. A consensus statement from the European Atherosclerosis Society Consensus Panel. Eur Heart J 38: 2459-2472, 2017.

2. Chen LY, Leening MJ, Norby FL, Roetker NS, Hofman A, Franco OH, Pan W, Polak JF, Witteman JC, Kronmal RA, et al: Carotid intima-media thickness and arterial stiffness and the risk of atrial fibrillation: The Atherosclerosis Risk in Communities (ARIC) Study, Multi-Ethnic Study of Atherosclerosis (MESA), and the Rotterdam Study. J Am Heart Assoc 5: e002907, 2016.

3. Trpkovic A, Resanovic I, Stanimirovic J, Radak D, Mousa SA, Cenic-Milosevic D, Jevremovic D and Isenovic ER: Oxidized low-density lipoprotein as a biomarker of cardiovascular diseases. Crit Rev Clin Lab Sci 52: 70-85, 2015.

4. Weber $\mathrm{C}$ and Noels H: Atherosclerosis: Current pathogenesis and therapeutic options. Nat Med 17: 1410-1422, 2011.

5. Mestas J and Ley K: Monocyte-endothelial cell interactions in the development of atherosclerosis. Trends Cardiovasc Med 18: 228-232, 2008

6. Koroleva IA, Nazarenko MS and Kucher AN: Role of microRNA in development of instability of atherosclerotic plaques. Biochemistry (Mosc) 82: 1380-1390, 2017.

7. Laffont B and Rayner KJ: MicroRNAs in the pathobiology and therapy of atherosclerosis. Can J Cardiol 33: 313-324, 2017.

8. Ambros V: The functions of animal microRNAs. Nature 431: 350-355, 2004.

9. Bartel DP: MicroRNAs: Genomics, biogenesis, mechanism, and function. Cell 116: 281-297, 2004.

10. Papageorgiou N, Tslamandris S, Giolis A and Tousoulis D: MicroRNAs in cardiovascular disease: Perspectives and reality. Cardiol Rev 24: 110-118, 2016.

11. Giral H, Kratzer A and Landmesser U: MicroRNAs in lipid metabolism and atherosclerosis. Best Pract Res Clin Endocrinol Metab 30: 665-676, 2016.

12. Matsushita R, Seki N, Chiyomaru T, Inoguchi S, Ishihara T, Goto Y, Nishikawa R, Mataki H, Tatarano S, Itesako T, et al: Tumour-suppressive microRNA-144-5p directly targets CCNE1/2 as potential prognostic markers in bladder cancer. Br J Cancer 113: 282-289, 2015.

13. Yamada Y, Arai T, Kojima S, Sugawara S, Kato M, Okato A, Yamazaki K, Naya Y, Ichikawa T and Seki N: Regulation of antitumor miR-144-5p targets oncogenes: Direct regulation of syndecan-3 and its clinical significance. Cancer Sci 109: 2919-2936, 2018.

14. Song L, Peng L, Hua S, Li X, Ma L, Jie J, Chen D, Wang Y and Li D: miR-144-5p Enhances the radiosensitivity of non-small-cell lung cancer cells via targeting ATF2. BioMed Res Int 2018: $5109497,2018$.

15. Li J, Wang R, Ge Y, Chen D, Wu B and Fang F: Assessment of microRNA-144-5p and its putative targets in inflamed gingiva from chronic periodontitis patients. J Periodontal Res 54: 266-277, 2019.

16. Wang X, Sundquist K, Hedelius A, Palmér K, Memon AA and Sundquist J: Circulating microRNA-144-5p is associated with depressive disorders. Clin Epigenetics 7: 69, 2015.

17. Goumans MJ, Liu Z and ten Dijke P: TGF-beta signaling in vascular biology and dysfunction. Cell Res 19: 116-127, 2009.

18. Bai Y, Zhang Q, Su Y, Pu Z and Li K: Modulation of the proliferation/apoptosis balance of cascular smooth muscle cells in atherosclerosis by lncRNA-MEG3 via regulation of miR-26a/smad1 axis. Int Heart J 60: 444-450, 2019.

19. Weng J, Wang C, Zhong W, Li B, Wang Z, Shao C, Chen Y and Yan J: Activation of CD137 signaling promotes angiogenesis in atherosclerosis via modulating endothelial Smad1/5-NFATc1 pathway. J Am Heart Assoc 6: 6, 2017.

20. Peng YG and Zhang L: Baohuoside-I suppresses cell proliferation and migration by up-regulating miR-144 in melanoma. Pharm Biol 56: 43-50, 2018.

21. Livak KJ and Schmittgen TD: Analysis of relative gene expression data using real-time quantitative PCR and the $2(-\Delta \Delta \mathrm{C}(\mathrm{T}))$ Method. Methods 25: 402-408, 2001. 
22. Mudau M, Genis A, Lochner A and Strijdom H: Endothelial dysfunction: The early predictor of atherosclerosis. Cardiovasc J Afr 23: 222-231, 2012

23. Li L, Li Y and Tang C: The role of microRNAs in the involvement of vascular smooth muscle cells in the development of atherosclerosis. Cell Biol Int 43: 1102-1112, 2019.

24. Su Y, Yuan J, Zhang F, Lei Q, Zhang T, Li K, Guo J, Hong Y, Bu G, Lv X, et al: MicroRNA-181a-5p and microRNA-181a-3p cooperatively restrict vascular inflammation and atherosclerosis. Cell Death Dis 10: 365, 2019.

25. Li K, Chen ZT and Qin YW: Expression profiles of microRNA related to atherosclerosis in patients with OSA. Lin Chung Er Bi Yan Hou Tou Jing Wai Ke Za Zhi 33: 304-309, 2019 (In Chinese).

26. Wang Z, Zhang J, Zhang S, Yan S, Wang Z, Wang C and Zhang X miR-30e and miR-92a are related to atherosclerosis by targeting ABCA1. Mol Med Rep 19: 3298-3304, 2019.

27. Cheng Y, Zhou M and Zhou W: MicroRNA-30e regulates TGF- $\beta$-mediated NADPH oxidase 4-dependent oxidative stress by Snai1 in atherosclerosis. Int J Mol Med 43: 1806-1816, 2019.

28. Yang L and Gao C: miR-590 inhibits endothelial cell apoptosis by inactivating the TLR4/NF- $\kappa \mathrm{B}$ pathway in atherosclerosis. Yonsei Med J 60: 298-307, 2019.

29. Fang M, Li Y, Wu Y, Ning Z, Wang X and Li X: miR-185 silencing promotes the progression of atherosclerosis via targeting stromal interaction molecule 1. Cell Cycle 18: 682-695, 2019.
30. Biedermann BC: Vascular endothelial cells: An interesting immunological barrier. Schweiz Med Wochenschr 129: 1712-1716, 1999 (In German).

31. Tian X, Yu C, Shi L, Li D, Chen X, Xia D, Zhou J, Xu W, Ma C, Gu L, et al: MicroRNA-199a-5p aggravates primary hypertension by damaging vascular endothelial cells through inhibition of autophagy and promotion of apoptosis. Exp Ther Med 16: 595-602, 2018

32. Soufi-Zomorrod M, Hajifathali A, Kouhkan F, Mehdizadeh M, Rad SM and Soleimani M: MicroRNAs modulating angiogenesis: miR-129-1 and miR-133 act as angio-miR in HUVECs. Tumour Biol 37: 9527-9534, 2016.

33. Liu F, Hata A, Baker JC, Doody J, Cárcamo J, Harland RM and Massagué J: A human Mad protein acting as a BMP-regulated transcriptional activator. Nature 381: 620-623, 1996.

34. Ren YF, Zhang TH, Zhong S, Zhao YT and Lv YN: miR-144 suppresses proliferation and induces apoptosis of osteosarcoma cells via direct regulation of mTOR expression. Oncol Lett 15: 1163-1169, 2018

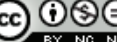

This work is licensed under a Creative Commons
Attribution-NonCommercial-NoDerivatives 4.0 International (CC BY-NC-ND 4.0) License. 